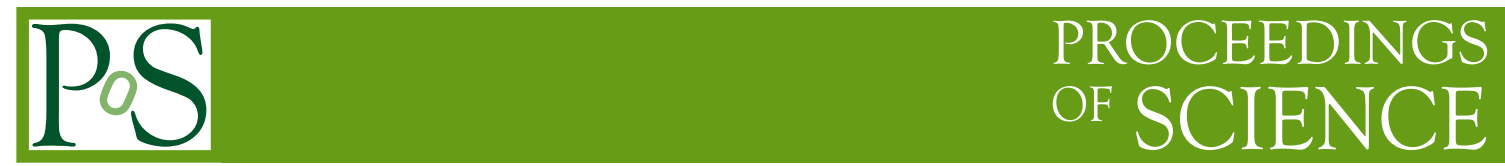

\title{
Inclusive radiative $B$ meson decays at Belle
}

\author{
Boštjan Golob*† \\ Jozef Stefan Institute, Ljubljana \\ E-mail: bostjan.golob@ijs.si
}

We present the most precise inclusive measurement of the branching fraction and photon energy spectrum of $b \rightarrow s \gamma$ radiative decays up to date. The photon energy distribution is measured for $E_{\gamma}>1.7 \mathrm{GeV}$. The measurement is performed by the Belle collaboration using $605 \mathrm{fb}^{-1}$ of data recorded by the Belle detector at the KEKB collider.

European Physical Society Europhysics Conference on High Energy Physics

July 16-22, 2009

Krakow, Poland

${ }^{*}$ Speaker.

${ }^{\dagger}$ Representing the Belle Collaboration 

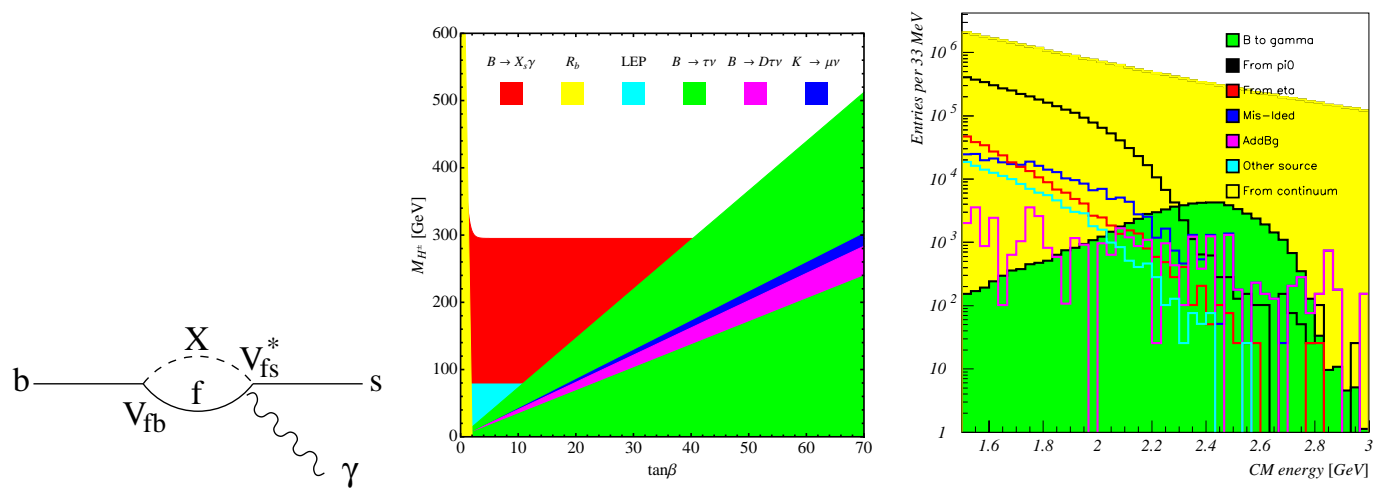

Figure 1: Left: Feynman diagram of $b \rightarrow s \gamma$ process where $X=W^{ \pm}$within the SM or some other particle in beyond the SM theories. Middle: Exclusion regions in the plane of charged Higgs boson parameters within the Type II Two Higgs Doublets Models arising from various measurements [1]. Right: Spectrum of photons from $b \rightarrow s \gamma$ signal and various sources of background.

\section{Introduction}

Radiative decays of $B$ mesons, where in the final state a hadronic system composed of an $s$ quark is found, have an underlying $b \rightarrow s \gamma$ quark process. Within the Standard Model (SM) such flavor changing neutral currents proceed through a loop diagram of Fig. 1 (left), where $X$ is a charged $W^{ \pm}$boson, $f$ is an uplike quark and $V_{f b}, V_{f s}$ are the appropriate elements of the CabibboKobayashi-Maskawa (CKM) matrix. As similar rare decays this process is sensitive to possible new processes and yet unobserved particles that could contribute in the loop. Examples include the charged Higgs bosons, $X=H^{ \pm}, f=u, c, t$, or charginos, $X=\chi^{ \pm}, f=\tilde{q}$. The rate induced by such New Physics (NP) processes depends on the corresponding couplings replacing the elements of the CKM matrix. Measurements of the $b \rightarrow s \gamma$ branching fraction impose constraints on the parameters of various extensions of the SM. An example is shown in Fig. 1 (middle) where the 95\% C.L. exclusion region in the mass of the charged Higgs boson and the Higgs vacuum expectation value is shown. The $\operatorname{Br}(b \rightarrow s \gamma)$ excludes the charged Higgs boson with $M_{H^{ \pm}} \leq 300 \mathrm{GeV}$ almost independently of $\tan \beta$ [1]. Furthermore, the shape of the photon spectrum in this process depends on the same non-perturbative QCD parameters as entering the expressions for the rate of semileptonic $B$ meson decays [2]. A precise determination of the $E_{\gamma}$ distribution can thus help in improving the accuracy of $\left|V_{c b}\right|$ and $\left|V_{u b}\right|$ determination. In the paper we present a recent measurement [3] of the $b \rightarrow s \gamma$ process using the data collected by the Belle detector [4] at the KEKB $e^{+} e^{-}$collider [5] with an integrated luminosity of $605 \mathrm{fb}^{-1}$.

\section{Measurement method}

The performed measurement is a fully inclusive one where only a photon arising from the signal $B$ meson is searched for in the detector. The other $B$ meson from the $\Upsilon(4 S)$ is either not used (untagged sample) or a lepton candidate consistent with a semileptonic decay of the tagging $B$ meson is reconstructed (tagged sample). The two reconstructed samples have a similar sensitivity 
to the $\operatorname{Br}\left(B \rightarrow X_{s} \gamma\right)$ as estimated using the simulation, and are to a large extent statistically independent (typical statistical correlation is found to be around 0.1). Hence the results obtained using the two samples can be averaged, improving the accuracy of the measurement.

The main experimental difficulty in the measurements of $B \rightarrow X_{s} \gamma$ is a large amount of background present at low photon energies. Hence the photon spectrum ${ }^{1}$ is measured for $E_{\gamma}$ above a certain lower cut-off value $E_{\gamma}^{\min }$. A theory based extrapolation associated with theoretical uncertainties is then required to obtain the full branching fraction. It is desirable to use as low value of $E_{\gamma}^{\min }$ as experimentally possible. The simulated $\gamma$ spectrum of signal and various sources of backgrounds is shown in Fig. 1 (right). By far the largest background source are photons from hadron decays arising from the continuum production of $u, d, s$ and $c$ quark pairs. At $E_{\gamma}^{\min }=1.8 \mathrm{GeV}$, the lowest cut-off value used so far in the existing measurements, the background from this source is around three orders of magnitude higher than the signal. At even lower value $E_{\gamma}^{\min }=1.7 \mathrm{GeV}$ used in this measurement the signal-to-noise ratio drops by additional 50\%. Data recorded at the collision energy around $60 \mathrm{MeV}$ below the $\Upsilon(4 S)$ represents a clean sample of such background photons. The integrated luminosity of this off-resonance data is $68 \mathrm{fb}^{-1}$. We subtract the scaled $E_{\gamma}$ distribution of the off-resonance data from the on-resonance data to obtain the spectrum of $\gamma$ 's from the $B$ meson decays:

$$
N^{B \bar{B}}\left(E_{\gamma}\right)=N^{O N}\left(E_{\gamma}\right)-\alpha C_{\varepsilon} N^{O F F}\left(F_{E} E_{\gamma}\right)
$$

where $\alpha$ is the luminosity scale factor including the $\sqrt{s}$ dependence of the cross-section, $C_{\varepsilon}$ is the correction factor taking into account a slight difference in the efficiency of the photon reconstruction between the off- and the on-resonance data, and $F_{E}$ is the correction factor arising from a small difference of the mean energy of photons in the two samples. The second largest source of background are two photon decays of neutral pions and $\eta$ mesons produced in $B$ meson decays. An explicit veto of $\gamma$ pairs with an invariant mass consistent with the $\pi^{0}$ or $\eta$ is used to suppress this type of background. After the selection the signal becomes the largest component of the sample. The remaining fraction of photons from light hadrons is subtracted using $E_{/}$distributions obtained from simulation and scaled according to the real data inclusive $B \rightarrow \pi^{0} X$ and $B \rightarrow \eta X$ control samples. The efficiency of the light hadron veto is determined using a partially reconstructed $D^{0} \rightarrow K^{-} \pi^{+} \pi^{0}$ data sample. The beam background distribution is obtained from the random trigger events.

\section{Results}

After the subtraction of backgrounds, scaled by appropriate factors obtained from the control samples, the measured $E_{\gamma}$ distribution is corrected for the selection efficiency ( $\sim 15 \%$ in the untagged and $\sim 2.5 \%$ in the tagged sample). The average of the spectra obtained in the tagged and untagged samples (taking stat. correlations into account) is presented in Fig. 2 (left).

The branching fraction is calculated by integrating the measured photon spectrum. A few steps are necessary to obtain the $\operatorname{Br}\left(B \rightarrow X_{s} \gamma\right)$ from the raw spectrum: unfolding of the photon energy (calibrated using radiative di-muon events; the average relative resolution on $E_{\gamma}$ is found to be

\footnotetext{
${ }^{1}$ The photon energy is taken in the center-of-mass system of the $e^{+} e^{-}$collision.
} 

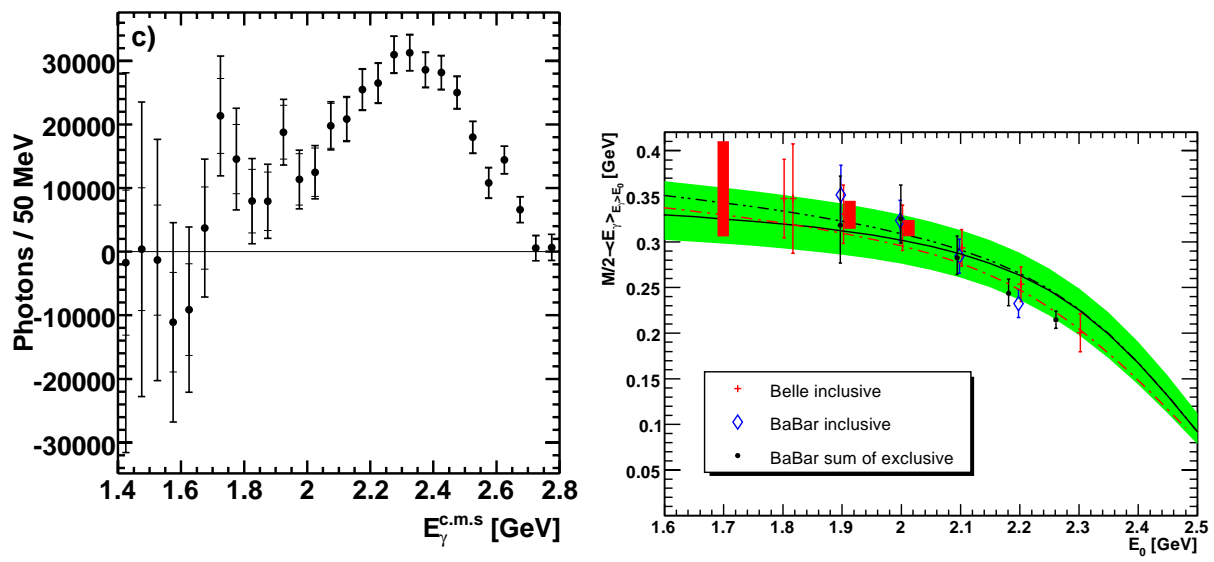

Figure 2: Left: The spectrum of photons from $b \rightarrow s \gamma$ as obtained by averaging the results in tagged and untagged sample. Right: $M_{B} / 2-\left\langle E_{\gamma}\right\rangle$ as obtained in this measurement (thick dark/red bar) as a function of $E_{\gamma}^{\min }$, compared to theory prediction (shaded/green band) [6] and other existing measurements.

around 2\%); correcting for the electromagnetic cluster detection efficiency in the electromagnetic calorimeter; correcting for the contribution of $b \rightarrow d \gamma$ decays ( $4.5 \pm 0.3 \%$ ); and finally, boosting $E_{\gamma}$ into the $B$ meson rest frame. The measured partial branching fraction for photons in the energy interval $1.7 \mathrm{GeV} \leq E_{\gamma} \leq 2.8 \mathrm{GeV}$ is

$$
\operatorname{Br}\left(B \rightarrow X_{s} \gamma ; 1.7 \mathrm{GeV} \leq E_{\gamma} \leq 2.8 \mathrm{GeV}\right)=(3.45 \pm 0.15 \pm 0.40) \cdot 10^{-4} .
$$

The main systematic uncertainties are due to the $B$ meson backgrounds other than $\pi^{0}$ and $\eta$, and the uncertainty of correction factors in the off-resonance data subtraction. These systematic errors can be reduced with a larger available data set.

From the measured $E_{\gamma}$ spectrum the first and second moments of the distribution are calculated as a function of the $E_{\gamma}^{\min }$. An example of $\left\langle E_{\gamma}\right\rangle$ is shown in Fig. 2 (right) and compared to recent theory calculations [6].

A naive average of older [7] and the presented measurements, scaled by the Heavy Flavour Averaging Group factors [8] to obtain the branching fraction for $E_{\gamma}^{\min }=1.6 \mathrm{GeV}$, where the comparison to theoretical predictions is made, results in $\operatorname{Br}\left(B \rightarrow X_{s} \gamma ; E_{\gamma} \geq 1.6 \mathrm{GeV}\right)=(3.57 \pm 0.24) \cdot 10^{-4}$. This is in agreement with the expectation within the SM [9], $B r^{\mathrm{SM}}\left(B \rightarrow X_{s} \gamma ; E_{\gamma} \geq 1.6 \mathrm{GeV}\right)=$ $(3.15 \pm 0.23) \cdot 10^{-4}$.

\section{References}

[1] U. Haisch, arXiv:0805.2141.

[2] D. Benson et al., Nucl. Phys. B665, 367 (2003).

[3] A. Limosani et al. (Belle Coll.), arXiv:0907.1384.

[4] A. Abashian et al. (Belle Coll.), Nucl. Instr. Meth. A479, 117 (2002).

[5] S. Kurokawa, E. Kikutani, Nucl. Instr. Meth. A499, 1 (2003), and other papers in this Volume. 
[6] J.R. Andersen, E. Gardi, JHEP 0701, 029 (2007).

[7] S. Chen et al. (Celo Coll.), Phys. Rev. Lett. 87, 251807 (2001); K. Abe et al. (Belle Coll.), Phys. Lett. B511, 151 (2001); B. Aubert et al. (BaBar Coll.), Phys. Rev. D72, 052004 (2005); B. Aubert et al. (BaBar Coll.), Phys. Rev. Lett. 97, 171803 (2006); B. Aubert et al. (BaBar Coll.), Phys. Rev. D77, 051103 (2008).

[8] E. Barberio et al. (HFAG Group), arXiv:0808.1297.

[9] M. Misiak et al., Phys. Rev. Lett. 98, 022002 (2007). 